Mathematical Modelling and Analysis

Volume 22 Number 6, November 2017, 827-851

https://doi.org/10.3846/13926292.2017.1386239

(c) Vilnius Gediminas Technical University, 2017
Publisher: Taylor\&Francis and VGTU

http://www.tandfonline.com/TMMA

ISSN: $1392-6292$

eISSN: 1648-3510

\title{
Water-waves Scattering by Permeable Bottom in Two-layer Fluid in the Presence of Surface Tension
}

\author{
Srikumar Panda ${ }^{a}$ and Subash C. Martha ${ }^{b}$
}

${ }^{a}$ Department of Mathematics, Vidyasagar College 700006 Kolkata, India

${ }^{b}$ Department of Mathematics, Indian Institute of Technology Ropar

Nangal Road, 140001 Rupnagar, Punjab, India

E-mail(corresp.): shree.iitg.mc@gmail.com

Received January 9, 2017; revised September 22, 2017; published online November 15, 2017

\begin{abstract}
In the present paper, reflection and transmission phenomena of water waves due to undulating permeable bottom in a two-layer fluid system are investigated using two-dimensional linearized theory. The effect of surface tension on the free surface is included in this work. In two-layer fluid system, there exist waves with two different wave numbers (modes). When a wave of a particular wave number encounters the undulating bottom, reflection and transmission phenomena occur in both the layers. The reflection and transmission coefficients in both layers due to incident waves of both modes are analyzed with the aid of perturbation analysis along with Fourier transform technique. It is found that these coefficients are obtained in terms of integrals which depend on the shape function of the undulating bottom. Two different kinds of undulating bottoms are considered to determine these coefficients. For a particular undulating bottom, namely sinusoidal bottom undulation the effect of various physical parameters such as number of ripples, surface tension and porous effect parameters are demonstrated graphically. The study further elaborates the energy balance relations associated with the reflection and transmission coefficients to ascertain the correctness of all the computed results.
\end{abstract}

Keywords: two-layer fluid, porosity, surface tension, linear theory, wave scattering.

AMS Subject Classification: 35Q35; 76B15.

\section{Introduction}

In recent times, many researchers have shown their interest in the field of scattering of water waves in a multi-layered fluid system instead of a single layer fluid system. The growing interest is mainly due to the following facts: (i) a single layer approximation becomes insufficient as the fluid is continuously stratified; and (ii) the possibility of transformation of water wave energy from 
one mode to another mode. The propagation of waves in a two-layer fluid without the presence of any obstacle was first investigated by Stokes. For such a two-layer fluid system, there exist two possible linear wave systems [15] at a given frequency each with a different wave number (mode): waves with lower wave number propagate in the upper layer while those with higher wave number propagate in the lower layer. When a wave train of a particular mode interacts with an obstacle, it may happen that the incident wave is partially reflected and also partially transmitted into waves of both modes. Thus, there is a possibility that some of the incident wave energy may transfer from one mode to another. In this context, Linton and McIver [15] studied the problem involving scattering of water waves in the presence of a long horizontal cylinder present in either of the two layers in a two-layer fluid. Based on multipole expansion, they have obtained the reflection and transmission coefficients for both wave numbers due to incident wave of two modes. Subsequently, Linton and Cadby [14] have extended the problem of Linton and McIver [15] to the scattering of oblique waves. They have also studied the reflection and transmission phenomena with the help of multipole expansion method. Afterwards, Chamberlain and Porter [8] have studied the problem involving scattering of water waves in a two-layer fluid of varying depth. It should be noted that, these kind of problems arise while modelling an underwater pipe bridge across one of the Norwegian fjords which consists of a layer of fresh water on top of a deep layer of salt water.

The scattering of water waves in a two-layer fluid over an obstacle or a geometrical disturbance at the bottom has been considered for its possible applications in the areas of coastal and marine engineering. The problem involving reflection and transmission of waves by patches of bottom undulation has received an increasing attention in recent time since its mechanism is important in the development of shore parallel bars or pipes. The problem involving scattering of water waves in single layer fluid was studied by Davies [10], Davies and Heathershaw [11], Martha et al. [4] and many others by employing various mathematical techniques. In case of two-layer fluid, the scattering of water waves by small bottom undulation was studied by Maiti and Mandal [16], Panda [21], Mohapatra and Bora [19]- [20] and references therein. They have employed different mathematical techniques to solve the respective problems. In all the above studies, the wave motion in the water region is investigated excluding the effect of surface tension and porosity of the bottom. In reality, the porosity of the bottom becomes an exceedingly important aspect of coastal engineering as reported in the literature. Recently, the wave scattering by porous bottom has gained considerable interest. The effect of porosity of bottom in a single layer fluid system is well documented in literature [2] and references therein.

All the above works were focused only on the solutions of the water wave interaction problems by excluding the effect of surface tension. The study involving water waves in the presence of surface tension in a single layer fluid has been made by many researchers. Rhodes-Robinson [24] developed an effective reduction technique to study the effect of surface tension on the progressive waves due to vertical wave makers. Chakrabarti and Sahoo [7] studied the 
effect of surface tension in a single layer fluid using mixed-type Fourier transform in the case of infinite depth and the method of eigenfunction expansion in the case of finite depth. Using Fourier transform technique, Panda et al. [25] studied the scattering of water waves in a single layer fluid over a permeable bottom by considering the effect of surface tension. Harter et al. [1] analyzed the effect of surface tension on trapped modes. They pointed out that the exclusion of surface tension during the formulation of the problem is not always justifiable, as its inclusion changes the qualitative nature of the streamline pattern. They also mentioned that the surface tension does not always play a qualitatively irrelevant role in the linear water-wave problem. Therefore, they advise to consider surface tension in any future works, as its inclusion could bring about unexpected results.

To the authors' knowledge, the effect of surface tension at the free surface in a two-layer fluid is yet to be known. In present paper, scattering of water waves by undulating permeable bottom in a two-layer fluid system in the presence of surface tension at the free surface is studied. It is assumed that the fluid in each layer is incompressible and inviscid; and the flow is irrotational. In two-layer fluid system, the time-harmonic waves of a particular mode can propagate with two-different wave numbers: waves with lower wave number propagate in the upper layer and the waves with higher wave number propagate in the lower layer. Using linear theory, the problem under consideration is formulated as a coupled boundary value problem (BVP) for two velocity potential functions describing the fluid motion in each layer. Employing perturbation analysis, the velocity potentials and the reflection as well as transmission coefficients appearing in the coupled BVP can be expanded as a power series of the parameter $\varepsilon$ representing smallness of the undulation. After equating the coefficients of the identical powers of $\varepsilon$ from both sides of all equations of the coupled BVP, a number of BVPs can be obtained. However, the BVPs up to first order are considered here for their solutions as the BVPs of higher order can be solved in this principle successively. The solution of the first order BVP is derived using Fourier transform technique. This solution is utilized to determine the first order reflection and transmission coefficients which are obtained in terms of integrals involving the shape function of the undulating bottom. Two different kinds of undulating bottoms are considered to evaluate the reflection and transmission coefficients (physical quantities of interest) due to incident waves of both modes. In addition, the energy balance relations known as the energy identities involving reflection and transmission coefficients are derived in detail using modified Green's integral theorem to ascertain the correctness of all computed results.

\section{Mathematical formulation of the problem}

We have considered a two-layer fluid system as shown in Figure 1 in which the upper layer is free to the atmosphere and the bottom of the lower layer has a small undulation. In this study, we have also considered the effect of surface tension at the free surface and the porosity in the undulating bottom, which is an endeavour to take the problem to another step forward towards a 


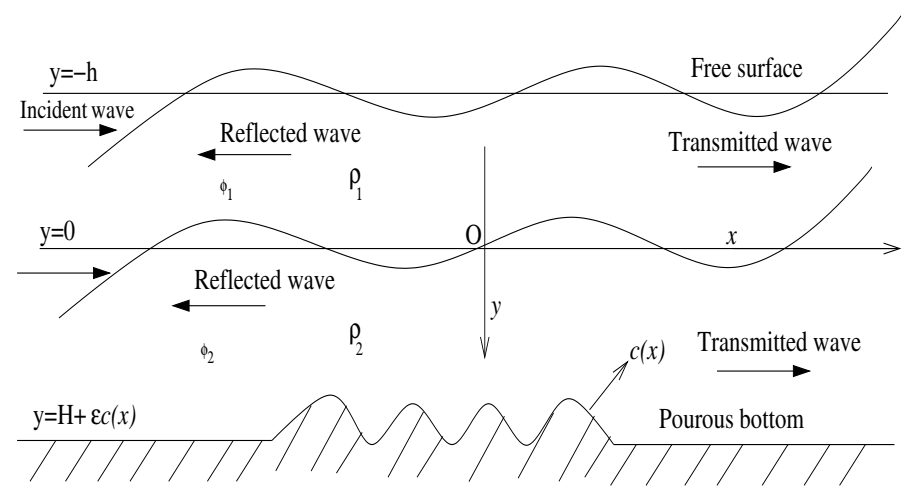

Figure 1. Schematic diagram

real and practical situation occurring in the water wave scattering problem. It is assumed that the motion to be irrotational and simple harmonic; and the fluid in each layer is inviscid, incompressible and immiscible having constant but different densities. The motion of the fluid inside the porous bed is not analyzed in this study. To formulate the problem mathematically, $x$ axis is taken along the undisturbed interface between the two fluids and the $y$ axis is taken vertically downward from the undisturbed interface. The fluid in the upper layer occupies the region $-h \leq y \leq 0$, where $h$ is the mean position of the free surface. The fluid in the lower layer occupies the region $0 \leq y \leq$ $H+\varepsilon c(x)$, where $y=H+\varepsilon c(x)$ represents the undulating permeable bottom, $c(x)$ (bounded and continuous function) describing shape of the undulation, $\varepsilon$ is a small parameter giving a measure of the undulation, $H$ is uniform finite depth far to either side of the undulation so that $c(x) \rightarrow 0$ as $|x| \rightarrow \infty$. The density of the fluid in the upper layer is denoted by $\rho_{1}$ whereas the density of the lower layer fluid is denoted by $\rho_{2}\left(>\rho_{1}\right)$. Under the assumptions as mentioned above, velocity potentials in the upper and lower layer can respectively be written as

$$
\Phi_{1}(x, y, \tilde{t})=\operatorname{Re}\left[\phi_{1}(x, y) e^{-i \sigma \tilde{t}}\right], \quad \Phi_{2}(x, y, \tilde{t})=\operatorname{Re}\left[\phi_{2}(x, y) e^{-i \sigma \tilde{t}}\right],
$$

where $\sigma$ is the angular frequency of the incoming waves and $\tilde{t}$ is the time. In each layer, the equation of continuity yields the Laplace equation ( $[15])$ :

$$
\begin{array}{ll}
\nabla^{2} \phi_{1}=0, & \infty<x<\infty, \quad-h \leq y \leq 0, \\
\nabla^{2} \phi_{2}=0, & \infty<x<\infty, \quad 0 \leq y \leq H+\varepsilon c(x),
\end{array}
$$

where $\nabla^{2}$ is the two-dimensional Laplace operator.

The linearized condition at the free surface with the effect of surface tension can be written as ([24]) mentioned below

$$
\left(\phi_{1}\right)_{y}+K \phi_{1}+M\left(\phi_{1}\right)_{y y y}=0, \quad \text { on } \quad y=-h,
$$

where $K=\frac{\sigma^{2}}{g}$ with $g$ being acceleration due to gravity and $M=\frac{S}{\rho_{1} g}$ with $S$ is the coefficient of surface tension. Here it should be noted that the dimension of $K$ is (length) $)^{-1}$ and the dimension of $M$ is (length $)^{2}$. 
The linearized interface conditions ( [20]) are

$$
\begin{aligned}
\left(\phi_{1}\right)_{y} & =\left(\phi_{2}\right)_{y}, & & \text { on } \quad y=0, \\
\rho\left[\left(\phi_{1}\right)_{y}+K \phi_{1}\right] & =\left(\phi_{2}\right)_{y}+K \phi_{2}, & & \text { on } \quad y=0,
\end{aligned}
$$

where $\rho=\frac{\rho_{1}}{\rho_{2}}(<1)$. The boundary condition (2.4) is due to the continuity of normal velocity whereas the condition (2.5) is due to the continuity of pressure at the interface.

Based on the assumption made by Darcy, the condition at the porous bottom can be written as $([3,4,9])$

$$
\frac{\partial \phi_{2}}{\partial n}-G \phi_{2}=0, \quad \text { on } \quad y=H+\varepsilon c(x),
$$

where $\partial / \partial n$ denotes the normal derivative at a point $(x, y)$ on the bottom and $G$ is the porous effect parameter $([4,9,17])$ with dimension of $G$ is length $^{-1}$ corresponding to the undulating bottom under consideration. Here we assume that the ocean bed is composed of specific types of porous materials with very fine pores. Therefore, the boundary condition given in relation (2.6) is valid throughout the study. It should be noted that the porous boundary condition (2.6) is an ad hoc boundary condition which takes up the rigid boundary condition when the porous parameter $G$ tends to zero. The value of the porous parameter $G$ is chosen as real throughout the study. The value of $G$ can be choosen to be real due to the percolation at the fluid-porous interface in which only flow resistance is considered. It is worth mentioning that the progressive waves exist for real value of $G$ only. However, if the value of $G$ is complex, there does not exist any progressive waves. The condition (2.6) is due to the porous beds, like a sand bed or any other non-rigid bed through which water can pass through. Such an assumption of non-rigid and porous bed induces boundary condition of the impedance type involving a linear combination of the potential function and its normal derivative on the boundary for the case of irrotational flow as it has been used in this study.

The bottom boundary condition (2.6) can be approximated as

$$
\left(\phi_{2}\right)_{y}-\varepsilon \frac{\partial}{\partial x}\left[c(x)\left(\phi_{2}\right)_{x}\right]-G\left[\phi_{2}+\varepsilon c(x)\left(\phi_{2}\right)_{y}\right]+\mathrm{O}\left(\varepsilon^{2}\right)=0, \quad \text { on } \quad y=H .
$$

In addition to the relations $(2.1)-(2.7)$, there are additional boundary conditions, namely the far-field conditions at $x \rightarrow \pm \infty$. These boundary conditions depend on the forms of the progressive waves. The forms of the progressive waves in the two-layer fluid associated with the given problem are derived by solving Laplace equations (2.1) and (2.2) along with the boundary conditions (2.3)-(2.5) and the flat bed condition $\partial \phi_{2} / \partial y-G \phi_{2}=0$, on $y=H$, using the method of separation of variables. The final expression of the progressive waves are obtained as follows:

$$
\begin{aligned}
& \phi_{10}(x, y)=f_{1}(k, y) e^{ \pm i k x}, \quad-\infty<x<\infty,-h \leq y \leq 0 \\
& \phi_{20}(x, y)=f_{2}(k, y) e^{ \pm i k x}, \quad-\infty<x<\infty, 0 \leq y \leq H
\end{aligned}
$$


where

$$
f_{1}(k, y)=\frac{\left[\sinh k H-\frac{G}{k} \sinh k H\right]\left[\left(1+M k^{2}\right) k \cosh k(h+y)-K \sinh k(h+y)\right]}{K \cosh k h-\left(1+M k^{2}\right) k \sinh k h}
$$

and

$$
f_{2}(k, y)=\left[\cosh k(H-y)-\frac{G}{k} \sinh k(H-y)\right]
$$

with $k$ satisfying the relation

$$
\Delta(k)=0,
$$

where

$$
\begin{aligned}
\Delta(k) & =k^{2}(1-\rho)\left(1+M k^{2}\right)-\frac{K^{2} G}{k}(\operatorname{coth} k h+\rho \operatorname{coth} k H) \\
& +K^{2}(\rho+\operatorname{coth} k h \operatorname{coth} k H)+G K\left[\left(1+M k^{2}\right)(1+\rho \operatorname{coth} k h \operatorname{coth} k H)\right. \\
& +(1-\rho) \operatorname{coth} k h \operatorname{coth} k H]-\left(1+M k^{2}\right) k G(1-\rho) \operatorname{coth} k H \\
& -K k\left[\left(1+M k^{2}\right)(\rho \operatorname{coth} k h+\operatorname{coth} k H)+(1-\rho) \operatorname{coth} k h\right] .
\end{aligned}
$$

The eigenfunctions $f_{1}(k, y)$ and $f_{2}(k, y)$ defined in relations $(2.10)-(2.11)$ are integrable in $(-h<y<0) \cup(0<y<H)$ having a single discontinuity at $y=0$ and are orthogonal with respect to the inner product as defined (by applying similar analysis of [13]) by

$$
<\phi, \psi>=\rho \int_{-h}^{0} \phi(y) \psi(y) d y+\int_{0}^{H} \phi(y) \psi(y) d y .
$$

Here it is worthy to mention that the functions $f_{1}(k, y)$ and $f_{2}(k, y)$ are the eigenfunctions associated with the self-adjoint operator

$$
L \Theta \equiv \frac{d^{2} \Theta}{d y^{2}}=\lambda^{2} \Theta, \quad y \in(-h, 0) \cup(0, H),
$$

corresponding to the eigenvalues $\lambda=\lambda_{1}, \lambda_{2}$ satisfying the end conditions

$$
\Theta^{\prime}(-h)+K \Theta(-h)+M \Theta^{\prime \prime \prime}(-h)=0 \quad \text { and } \quad \Theta^{\prime}(H)-G \Theta(H)=0
$$

and the interface condition

$$
\Theta^{\prime}\left(0^{-}\right)=\Theta^{\prime}\left(0^{+}\right) \quad \text { and } \quad \rho_{1}\left[\Theta^{\prime}\left(0^{-}\right)+K \Theta\left(0^{-}\right)\right]=\rho_{2}\left[\Theta^{\prime}\left(0^{+}\right)+K \Theta\left(0^{+}\right)\right] \text {, }
$$

where ' denotes the first order derivative.

The relation (2.12) is called as dispersion relation in the theory of water wave. It can be shown (discussed later in Section 6) that the dispersion relation (2.12) has two positive real roots (say, $\lambda_{1}$ and $\lambda_{2}$ with $\lambda_{1}<\lambda_{2}$ ) which indicate that there exist incident (progressive) waves of two different modes (wave numbers) $\lambda_{1}$ and $\lambda_{2}$. Let us first consider the wave of mode $\lambda_{1}$ is incident from the direction $x=-\infty$ to the bottom undulation then it is partially reflected 
and partially transmitted into waves in both layers. Therefore, the far-field conditions can be expressed as

$$
\phi_{1}(x, y) \rightarrow \begin{cases}f_{1}\left(\lambda_{1}, y\right)\left(e^{i \lambda_{1} x}+r e^{-i \lambda_{1} x}\right)+R f_{1}\left(\lambda_{2}, y\right) e^{-i \lambda_{2} x}, & x \rightarrow-\infty \\ t f_{1}\left(\lambda_{1}, y\right) e^{i \lambda_{1} x}+T f_{1}\left(\lambda_{2}, y\right) e^{i \lambda_{2} x}, & x \rightarrow \infty\end{cases}
$$

and

$$
\phi_{2}(x, y) \rightarrow \begin{cases}f_{2}\left(\lambda_{1}, y\right)\left(e^{i \lambda_{1} x}+r e^{-i \lambda_{1} x}\right)+R f_{2}\left(\lambda_{2}, y\right) e^{-i \lambda_{2} x}, & x \rightarrow-\infty, \\ t f_{2}\left(\lambda_{1}, y\right) e^{i \lambda_{1} x}+T f_{2}\left(\lambda_{2}, y\right) e^{i \lambda_{2} x}, & x \rightarrow \infty\end{cases}
$$

where $r, R$ are respectively the reflection coefficients associated with reflected waves of modes $\lambda_{1}$ and $\lambda_{2}$, due to incident wave of mode $\lambda_{1}$. Similarly, $t$ and $T$ denote the transmission coefficients associated with transmitted waves of modes $\lambda_{1}$ and $\lambda_{2}$ respectively, due to incident wave of mode $\lambda_{1}$. These unknown reflection and transmission coefficients $(r, R, t$ and $T$ ) can be determined by solving the BVP given by relations (2.1)-(2.5), (2.7), (2.13) and (2.14).

When the wave of mode $\lambda_{2}$ is incident then the far-field conditions are as follows:

$$
\phi_{1}(x, y) \rightarrow \begin{cases}f_{1}\left(\lambda_{2}, y\right)\left(e^{i \lambda_{2} x}+\widehat{R} e^{-i \lambda_{2} x}\right)+\widehat{r} f_{1}\left(\lambda_{1}, y\right) e^{-i \lambda_{1} x}, & x \rightarrow-\infty \\ \widehat{T} f_{1}\left(\lambda_{2}, y\right) e^{i \lambda_{2} x}+\widehat{t} f_{1}\left(\lambda_{1}, y\right) e^{i \lambda_{1} x}, & x \rightarrow \infty\end{cases}
$$

and

$$
\phi_{2}(x, y) \rightarrow \begin{cases}f_{2}\left(\lambda_{2}, y\right)\left(e^{i \lambda_{2} x}+\widehat{R} e^{-i \lambda_{2} x}\right)+\widehat{r} f_{2}\left(\lambda_{1}, y\right) e^{-i \lambda_{1} x}, & x \rightarrow-\infty \\ \widehat{T} f_{2}\left(\lambda_{2}, y\right) e^{i \lambda_{2} x}+\widehat{t} f_{2}\left(\lambda_{1}, y\right) e^{i \lambda_{1} x}, & x \rightarrow \infty\end{cases}
$$

where $\widehat{r}, \widehat{t}$ are respectively the reflection and transmission coefficients associated with reflected and transmitted waves of modes $\lambda_{1} ; \widehat{R}$ and $\widehat{T}$ are respectively the reflection and transmission coefficients associated with reflected and transmitted wave of mode $\lambda_{2}$ due to incident wave of mode $\lambda_{2}$. The unknown coefficients $\widehat{r}, \widehat{R}, \widehat{t}$ and $\widehat{T}$ can be obtained by solving the BVP given by relations $(2.1)-(2.5),(2.7),(2.15)$ and (2.16). The aim of the present work is the determination (analytically) of all these unknown coefficients $r, R, t, T, \widehat{r}, \widehat{R}, \widehat{t}$ and $\widehat{T}$ including the effects of various physical parameters on these coefficients.

\section{Solution of the problem}

This section is devoted to determine the velocity potential functions, reflection and transmission coefficients due to incident wave of both the modes. Let us consider the incident wave of mode $\lambda_{1}$ for which the physical quantities such as $r, R, t, T$ have to be determined. In a similar fashion, all the coefficients due to incident wave of mode $\lambda_{2}$ can also be determined.

The incident wave train will propagate without any hindrance if there is no undulation at the bottom. Hence, there will be transmission only. In view of this fact along with the approximated form of bottom condition (2.7), the 
velocity potentials $\phi_{1}, \phi_{2}$ and the quantities $t, T, r, R$ can be expressed in terms of the small parameter $\varepsilon$ as follows:

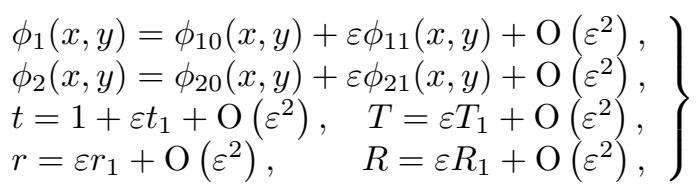

where $\phi_{10}(x, y)$ and $\phi_{20}(x, y)$ are given in relations (2.8) and (2.9) respectively and $\phi_{j 1}(j=1,2)$ denote the first order corrections of velocity potentials $\phi_{j}$. The reflection and transmission coefficients $r, R, t, T$ can be evaluated from the relation (3.1) once the first order coefficients $r_{1}, R_{1}, t_{1}, T_{1}$ are known. Hence, all these first order reflection and transmission coefficients are determined in this paper. It must be noted that such a perturbation analysis ceases to be valid at Bragg resonance when the reflection coefficient becomes much larger than the undulation parameter $\varepsilon$, as pointed out by Mei [18]. Since the reflection coefficient, considered in the present study, is not large as compared to the undulation parameter, therefore, the perturbation expansions as given in the relation (3.1) is valid throughout this paper.

Substituting the relation (3.1) in relations $(2.1)-(2.5),(2.7),(2.13),(2.14)$ and then comparing the first order terms of $\varepsilon$ on the both sides of all equations, we have the coupled BVP:

$$
\begin{aligned}
& \nabla^{2} \phi_{11}=0 \\
& -h \leq y \leq 0 \\
& \nabla^{2} \phi_{21}=0 \text {, } \\
& 0 \leq y \leq H \text {, } \\
& \left(\phi_{11}\right)_{y}+K \phi_{11}+M\left(\phi_{11}\right)_{y y y}=0 \text {, } \\
& \text { on } y=-h \text {, } \\
& \left(\phi_{11}\right)_{y}=\left(\phi_{21}\right)_{y} \text {, } \\
& \text { on } y=0 \text {, } \\
& \rho\left[\left(\phi_{11}\right)_{y}+K \phi_{11}\right]=\left(\phi_{21}\right)_{y}+K \phi_{21} \text {, } \\
& \text { on } y=0 \text {, } \\
& \left(\phi_{21}\right)_{y}-G \phi_{21}=q(x) \text {, } \\
& \text { on } y=H \text {, }
\end{aligned}
$$

where

$$
q(x)=i \lambda_{1} \frac{d}{d x}\left[c(x) e^{i \lambda_{1} x}\right]+G^{2} c(x),
$$

along with the first order far-field conditions

$$
\begin{aligned}
& \phi_{11}(x, y) \rightarrow\left\{\begin{array}{ll}
r_{1} f_{1}\left(\lambda_{1}, y\right) e^{-i \lambda_{1} x}+R_{1} f_{1}\left(\lambda_{2}, y\right) e^{-i \lambda_{2} x}, & x \rightarrow-\infty, \\
t_{1} f_{1}\left(\lambda_{1}, y\right) e^{i \lambda_{1} x}+T_{1} f_{1}\left(\lambda_{2}, y\right) e^{i \lambda_{2} x}, & x \rightarrow \infty
\end{array},\right. \\
& \phi_{21}(x, y) \rightarrow \begin{cases}r_{1} f_{2}\left(\lambda_{1}, y\right) e^{-i \lambda_{1} x}+R_{1} f_{2}\left(\lambda_{2}, y\right) e^{-i \lambda_{2} x}, & x \rightarrow-\infty, \\
t_{1} f_{2}\left(\lambda_{1}, y\right) e^{i \lambda_{1} x}+T_{1} f_{2}\left(\lambda_{2}, y\right) e^{i \lambda_{2} x}, & x \rightarrow \infty\end{cases}
\end{aligned}
$$

In order to solve the coupled BVP described by relations (3.2a)-(3.2f) for the potentials $\phi_{11}(x, y)$ and $\phi_{21}(x, y)$, the BVP has been decoupled by

$$
\left(\phi_{11}\right)_{y}=p(x) \text { and }\left(\phi_{21}\right)_{y}=p(x), \quad \text { on } \quad y=0,
$$

where $p(x)$ is an unknown function. Now the decoupled BVP is solved here using Fourier transform technique. The main advantage of using Fourier transform technique is that it is much easier and simpler than the other method (for 
example, Green function technique) utilized to study the problem involving scattering of water waves by Maiti and Mandal [16]. The Fourier transforms $\bar{\phi}_{11}(k, y)$ and $\bar{\phi}_{21}(k, y)$ of $\phi_{11}(x, y)$ and $\phi_{21}(x, y)$ respectively, defined as follows:

$$
\bar{\phi}_{11}(k, y)=\int_{-\infty}^{\infty} \phi_{11}(x, y) e^{-i k x} d x ; \quad \bar{\phi}_{21}(k, y)=\int_{-\infty}^{\infty} \phi_{21}(x, y) e^{-i k x} d x
$$

with inverses

$$
\phi_{11}(x, y)=\frac{1}{2 \pi} \int_{-\infty}^{\infty} \bar{\phi}_{11}(k, y) e^{i k x} d k ; \quad \phi_{21}(x, y)=\frac{1}{2 \pi} \int_{-\infty}^{\infty} \bar{\phi}_{21}(k, y) e^{i k x} d k .
$$

Using Fourier transform and inverse Fourier transform as defined above, the solution of the BVP is obtained as

$$
\begin{array}{r}
\phi_{11}(x, y)=\frac{K}{2 \pi} \int_{0}^{\infty} \frac{\left\{K \sinh k(h+y)-\left(1+M k^{2}\right) k \cosh k(h+y)\right\}}{k \sinh k h \sinh k H \Delta(k)} \\
\times\left[\bar{q}(k) e^{i k x}+\bar{q}(-k) e^{-i k x}\right] d k
\end{array}
$$

and

$$
\begin{array}{r}
\phi_{21}(x, y)=\frac{1}{2 \pi} \int_{0}^{\infty} \frac{1}{k\left(\sinh k H-\frac{G}{k} \cosh k H\right)}[\cosh k y \\
\left.-\frac{K\left[K \operatorname{coth} k h-k\left(1+M k^{2}\right)\right]\left[\cosh k(H-y)-\frac{G}{k} \sinh k(H-y)\right]}{\sinh k H \Delta(k)}\right] \\
\times\left[\bar{q}(k) e^{i k x}+\bar{q}(-k) e^{-i k x}\right] d k
\end{array}
$$

where $\bar{p}(k)$ and $\bar{q}(k)$ are the Fourier transform of $p(x)$ and $q(x)$ respectively.

In order to determine the first order transmission coefficients $t_{1}$ and $T_{1}$ due to incident wave of mode $\lambda_{1}$, we have taken $x \rightarrow \infty$ in relation (3.5) and then compared the resultant integral with relation (3.3). The transmission coefficients $t_{1}$ and $T_{1}$ are obtained as follows:

$$
\begin{aligned}
& t_{1}=Q\left(\lambda_{1}\right) \times\left(G^{2}-\lambda_{1}^{2}\right) \times \int_{-\infty}^{\infty} c(x) d x \\
& T_{1}=Q\left(\lambda_{2}\right) \times\left(G^{2}-\lambda_{1} \lambda_{2}\right) \times \int_{-\infty}^{\infty} c(x) e^{-i\left(\lambda_{2}-\lambda_{1}\right) x} d x,
\end{aligned}
$$

where $Q(k)=\frac{i K\left\{\left(1+M k^{2}\right) k \sinh k h-K \cosh k h\right\}}{\sinh k h \sinh k H(k \sinh k H-G \cosh k H) \Delta^{\prime}(k)}, \quad k=\lambda_{1}, \quad \lambda_{2}$.

The first order reflection coefficients $r_{1}$ and $R_{1}$ are obtained after evaluating the integrals given in relations (3.5) or (3.6) for $x \rightarrow-\infty$, and then comparing the behaviour of $\phi_{11}(x, y)$ or $\phi_{21}(x, y)$ with relation (3.3) or (3.4). The expressions of the first order reflection coefficients are

$$
\begin{array}{r}
r_{1}=-Q\left(\lambda_{1}\right) \times\left(G^{2}-\lambda_{1}^{2}\right) \int_{-\infty}^{\infty} c(x) e^{2 i \lambda_{1} x} d x \\
R_{1}=-Q\left(\lambda_{2}\right) \times\left(G^{2}-\lambda_{1} \lambda_{2}\right) \int_{-\infty}^{\infty} c(x) e^{i\left(\lambda_{2}+\lambda_{1}\right) x} d x .
\end{array}
$$


When the incident wave is of mode $\lambda_{2}$, the first order reflection and transmission coefficients $\widehat{r}_{1}, \widehat{R}_{1}, \widehat{t}_{1}$ and $\widehat{T}_{1}$ can be determined by solving the BVP consisting of Laplace equations (2.1) and (2.2) along with boundary conditions (2.3)-(2.5), (2.7), (2.15) and (2.16) by using the same mathematical procedure as described above for the case of mode $\lambda_{1}$. The coefficients $\widehat{r}_{1}, \widehat{R}_{1}, \widehat{t}_{1}$ and $\widehat{T}_{1}$ are obtained as follows:

$$
\begin{aligned}
& \widehat{t}_{1}=Q\left(\lambda_{1}\right) \times\left(G^{2}-\lambda_{1} \lambda_{2}\right) \times \int_{-\infty}^{\infty} c(x) e^{i\left(\lambda_{2}-\lambda_{1}\right) x} d x \\
& \widehat{T}_{1}=Q\left(\lambda_{2}\right) \times\left(G^{2}-\lambda_{2}^{2}\right) \times \int_{-\infty}^{\infty} c(x) d x \\
& \widehat{r}_{1}=-Q\left(\lambda_{1}\right) \times\left(G^{2}-\lambda_{1} \lambda_{2}\right) \times \int_{-\infty}^{\infty} c(x) e^{i\left(\lambda_{2}+\lambda_{1}\right) x} d x \\
& \widehat{R}_{1}=-Q\left(\lambda_{2}\right) \times\left(G^{2}-\lambda_{2}^{2}\right) \times \int_{-\infty}^{\infty} c(x) e^{2 i \lambda_{2} x} d x
\end{aligned}
$$

It can be noticed from relations (3.7), (3.8), and (3.9)-(3.14) that all the coefficients $r_{1}, R_{1}, t_{1}, T_{1}, \widehat{r}_{1}, \widehat{R}_{1}, \widehat{t}_{1}$ and $\widehat{T}_{1}$ depend on the shape function $c(x)$ of the undulating bottom. Hence, two different kinds of bottom profiles are considered in Section 5 to evaluate all the integrals in relations (3.7), (3.8), and (3.9)-(3.14). Further, it is also noticed that the reflection and transmission coefficients also depend on the porous effect parameter $G$ and the surface tension effect parameter $M$.

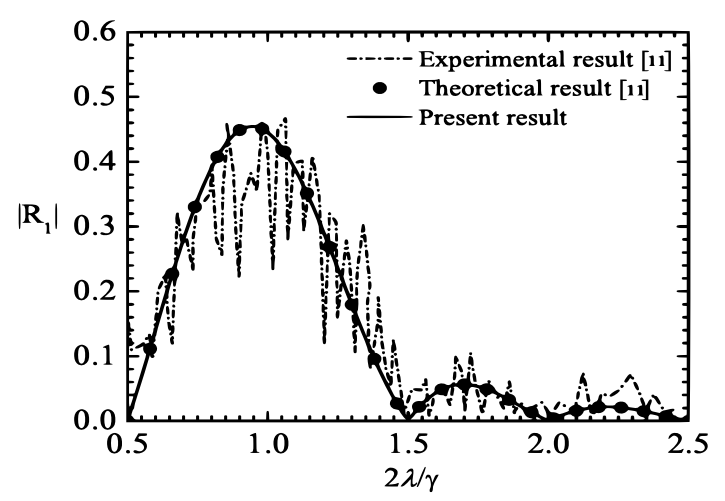

Figure 2. $\left|R_{1}\right|$ for $\rho_{1}=\rho_{2}, m=n=2, a /(h+H)=0.32, M / h^{2}=0, G h=0$.

\section{Validation of the results}

To validate the model under consideration, the present results are compared with the existing results available in the literature. To validate the present results we derive the results by considering $G=0$ (impermeable bottom) and $M=0$ (effect of surface tension is neglected) in relations (3.7), (3.8), and (3.9)-(3.14), the reflection and transmission coefficients are obtained. We have 
noticed that the derived results exactly match with the results of Mohapatra and Bora [19] (when $\theta=0$ in the absence of ice-sheet), Panda and Martha [23] (when $G=0$ and $\theta=0$ ), Panda and Martha [22] (when $G=0$ in the absence of ice-sheet).

In Figure 2, a comparison is made between the present results and the experimental as well theoretical results made by Davies and Heathershaw [11]. They performed the experiment on the problem involving scattering of water wave by irregular impermeable bottom having small undulation in a single layer. Therefore, we have derived the reflection coefficient, which is plotted in Figure 2 by considering $\rho_{1}=\rho_{2}$ and non-dimensional parameters $G h=$ $0, M / h^{2}=0$ in the present results. From Figure 2, it is clear that the results of the present study for $\rho_{1}=\rho_{2}$ are in good agreement with the theoretical as well as the experimental results of Davies and Heathershaw [11].

\section{Examples of undulating bottom profiles}

In this section we have considered different kinds of bottom profiles to demonstrate the influence of natural variation of bottom topography on the behaviour of reflection and transmission. The bottom profiles considered in the following section resemble with certain hump which are found in practical situations.

Example 1. Consider the following shape function $c(x)$ which corresponds to an exponentially damped undulating bottom,

$$
c(x)=b e^{-a_{0}|x|}\left(a_{0}>0\right),-\infty<x<\infty .
$$

Substituting relation (5.1) into relations (3.7), (3.8), (3.9), (3.10) and (3.11)(3.14), the first order reflection and transmission coefficients are obtained as

$$
\begin{aligned}
r_{1} & =-Q\left(\lambda_{1}\right) \times\left(G^{2}-\lambda_{1}^{2}\right) \times 2 b a_{0} /\left(a_{0}^{2}+4 \lambda_{1}^{2}\right), \\
R_{1} & =-Q\left(\lambda_{2}\right) \times\left(G^{2}-\lambda_{1} \lambda_{2}\right) \times 2 b a_{0} /\left(a_{0}^{2}+\left(\lambda_{2}+\lambda_{1}\right)^{2}\right), \\
t_{1} & =Q\left(\lambda_{1}\right) \times\left(G^{2}-\lambda_{1}^{2}\right) \times 2 b / a_{0}, \\
T_{1} & =Q\left(\lambda_{2}\right) \times\left(G^{2}-\lambda_{1} \lambda_{2}\right) \times \frac{2 b a_{0}}{a_{0}^{2}+\left(\lambda_{2}-\lambda_{1}\right)^{2}}, \\
\widehat{T}_{1} & =Q\left(\lambda_{2}\right) \times\left(G^{2}-\lambda_{2}^{2}\right) \times 2 b / a_{0}, \\
\widehat{t}_{1} & =Q\left(\lambda_{1}\right) \times\left(G^{2}-\lambda_{1} \lambda_{2}\right) \times \frac{2 b a_{0}}{a_{0}^{2}+\left(\lambda_{2}-\lambda_{1}\right)^{2}}, \\
\widehat{r}_{1} & =-Q\left(\lambda_{1}\right) \times\left(G^{2}-\lambda_{1} \lambda_{2}\right) \times \frac{2 b a_{0}}{a_{0}^{2}+\left(\lambda_{2}+\lambda_{1}\right)^{2}}, \\
\widehat{R}_{1} & =-Q\left(\lambda_{2}\right) \times\left(G^{2}-\lambda_{2}^{2}\right) \times 2 b a_{0} /\left(a_{0}^{2}+4 \lambda_{2}^{2}\right) .
\end{aligned}
$$

Example 2. Let us consider the shape function $c(x)$ in the form of a patch of sinusoidal ripples as the bottom undulation,

$$
c(x)= \begin{cases}a \sin \gamma x, & L_{1} \leq x \leq L_{2} \\ 0, & \text { otherwise }\end{cases}
$$


with $L_{1}=-n \pi / \gamma$ and $L_{2}=m \pi / \gamma$, where $m, n$ are positive integers. This represents a patch of sinusoidal ripples on an otherwise flat bottom. This patch consists total $(m+n) / 2$ ripples having the same wave number $\gamma$ and amplitude $a$. For this undulating bottom, all the reflection and transmission coefficients are derived as

$$
\begin{aligned}
& r_{1}=\frac{a \gamma\left(G^{2}-\lambda_{1}^{2}\right) Q\left(\lambda_{1}\right)}{\gamma^{2}-4 \lambda_{1}^{2}}\left[(-1)^{m} e^{2 i \lambda_{1} L_{2}}-(-1)^{n} e^{2 i \lambda_{1} L_{1}}\right] \\
& R_{1}=\frac{a \gamma\left(G^{2}-\lambda_{1} \lambda_{2}\right) Q\left(\lambda_{2}\right)}{\gamma^{2}-\left(\lambda_{1}+\lambda_{2}\right)^{2}}\left[(-1)^{m} e^{i\left(\lambda_{1}+\lambda_{2}\right) L_{2}}-(-1)^{n} e^{i\left(\lambda_{1}+\lambda_{2}\right) L_{1}}\right], \\
& T_{1}=\frac{a \gamma\left(G^{2}-\lambda_{1} \lambda_{2}\right) Q\left(\lambda_{2}\right)}{\gamma^{2}-\left(\lambda_{2}-\lambda_{1}\right)^{2}}\left[(-1)^{n} e^{i\left(\lambda_{1}-\lambda_{2}\right) L_{1}}-(-1)^{m} e^{i\left(\lambda_{1}-\lambda_{2}\right) L_{2}}\right], \\
& t_{1}=Q\left(\lambda_{1}\right) \times\left(G^{2}-\lambda_{1}^{2}\right) \times \frac{a}{\gamma}\left[(-1)^{n}-(-1)^{m}\right] \\
& \widehat{T}_{1}=Q\left(\lambda_{2}\right) \times\left(G^{2}-\lambda_{2}^{2}\right) \times \frac{a}{\gamma}\left[(-1)^{n}-(-1)^{m}\right] \\
& \widehat{t}_{1}=\frac{a \gamma\left(G^{2}-\lambda_{1} \lambda_{2}\right) Q\left(\lambda_{1}\right)}{\gamma^{2}-\left(\lambda_{2}-\lambda_{1}\right)^{2}}\left[(-1)^{n} e^{i\left(\lambda_{2}-\lambda_{1}\right) L_{1}}-(-1)^{m} e^{i\left(\lambda_{2}-\lambda_{1}\right) L_{2}}\right] \\
& \widehat{r}_{1}=\frac{a \gamma\left(G^{2}-\lambda_{1} \lambda_{2}\right) Q\left(\lambda_{1}\right)}{\gamma^{2}-\left(\lambda_{2}+\lambda_{1}\right)^{2}}\left[(-1)^{m} e^{i\left(\lambda_{2}+\lambda_{1}\right) L_{2}}-(-1)^{n} e^{i\left(\lambda_{2}+\lambda_{1}\right) L_{1}}\right] \\
& \widehat{R}_{1}=\frac{a \gamma\left(G^{2}-\lambda_{2}^{2}\right) Q\left(\lambda_{2}\right)}{\gamma^{2}-4 \lambda_{2}^{2}}\left[(-1)^{n} e^{2 i \lambda_{2} L_{1}}-(-1)^{m} e^{2 i \lambda_{2} L_{2}}\right] .
\end{aligned}
$$

\section{Results and discussion}

This section contains a discussion on the roots of the dispersion relation (2.12), the effect of various dimensionless system parameters on the reflection and transmission coefficients. This section also illustrate the nature of the free surface elevation as well as the interface elevation. Further, "energy identities", an important feature of the study involving scattering of water waves in a twolayer fluid are derived here by using modified Green's integral theorem. Out of two different kinds of bottom profiles considered in Section 5 to evaluate the reflection and transmission coefficients, the undulating bottom involving a patch of sinusoidal ripples (Example-2) is considered here to demonstrate the numerical results. The relevance of such consideration is mainly due to the following associated significance: (a) the sinusoidal bottom has the ability to reflect the incident wave energy which is important in coastal protection [10], and (b) the sinusoidal bottom undulation closely resembles some naturally occurring obstacle formed at the bottom due to sedimentation and ripple growth of sands. Hence, we fix our attention to compute the numerical results of the coefficients given by relations $(5.3)-(5.10)$.

Relations (5.3)-(5.5) and (5.8)-(5.10) illustrate that the first order reflection and transmission coefficients due to incident wave of both modes, are oscillatory in nature and assume zero values for several values of $K h$. In addition, it can also be realized from relations (5.3)-(5.5) and (5.8)-(5.10), at the critical conditions $\left(\gamma=2 \lambda_{1}, \gamma=\lambda_{2}+\lambda_{1}, \gamma=\lambda_{2}-\lambda_{1}\right.$ and $\left.\gamma=2 \lambda_{2}\right)$, a resonant 
interaction takes place between the undulating bottom and the incident wave. When $m=n$ in relation (5.2), i.e. the patch consists total $n$ ripples, then the transmission coefficients $t_{1}$ and $\widehat{T}_{1}$ (refer Eqs. (5.6) and (5.7)) vanish because $c(x)$ becomes an odd function. Several computations are performed and, in particular, some special results are tabulated and presented in graphical forms in the following section. These graphical forms for the reflection and transmission coefficients illustrate the role of various system parameters (number of ripples of the undulating bed, porosity, surface tension) on the transformation of wave energy between the waves of two modes.

All parameters are made dimensionless by using $h$ as length scale and therefore, the parameters $K h, G h, M / h^{2}, a / h, H / h$ and $\gamma h$ are all now become dimensionless quantities. For computational results shown in the present study, the values of various dimensionless physical parameters are kept fixed as depth ratio $H / h=2$, time $\tilde{t}=0$, amplitude of the sinusoidal ripples $a / h=0.1$, ripple wave number $\gamma h=1$, number of the ripples $n=3, m=5$, porous effect parameter $G h=0.05$ and surface tension parameter $M / h^{2}=0.2$, unless it is mentioned. Value of the density ratio $\rho$ is chosen $\rho=0.5$ as considered in [14].

\subsection{Roots of the dispersion relation}

The real positive roots of the dispersion relation (2.12) are calculated using Newton-Raphson method and are shown in Table 1 for different combinations of surface tension parameter $\left(M / h^{2}\right), K h$, density ratio $(\rho)$ and porous effect parameter $(G h)$.

Table 1. Roots $(k)$ of the dispersion relation (2.12) for different values of various parameters

\begin{tabular}{lll}
\hline Values of the parameters & Roots: (two-layer fluid) & Root: (single layer fluid) \\
\hline$K h=0.2, G h=0.04, M / h^{2}=0.2$ & $\begin{array}{l}0.31895 \\
0.81245\end{array}$ & 0.30244 \\
\hline$K h=0.6, G h=0.04, M / h^{2}=0.2$ & $\begin{array}{l}0.61542 \\
1.84733\end{array}$ & 0.59692 \\
\hline$K h=0.2, G h=0.06, M / h^{2}=0.2$ & 0.32839 & 0.81348 \\
\hline$K h=0.2, G h=0.04, M / h^{2}=0.5$ & 0.31534 & 0.80338 \\
\hline$\rho$
\end{tabular}

From Table 1, it is clear that the dispersion relation (2.12) has two non-zero real positive roots which ensures the remark reported in Section 2. In order to illustrate the behaviors of the roots of the dispersion relation (2.12), the contour plot of the relation (2.12) is depicted in Figure 3.

The contour plot shown in Figure 3 demonstrates the existence of two positive real roots $\left(\lambda_{1}\right.$ and $\left.\lambda_{2}\right)$ corresponding to two wave modes in the $k$-plane. Here, it should be noted that the roots $\lambda_{-1}$ and $\lambda_{-2}$ shown in Figure 3 refer to 


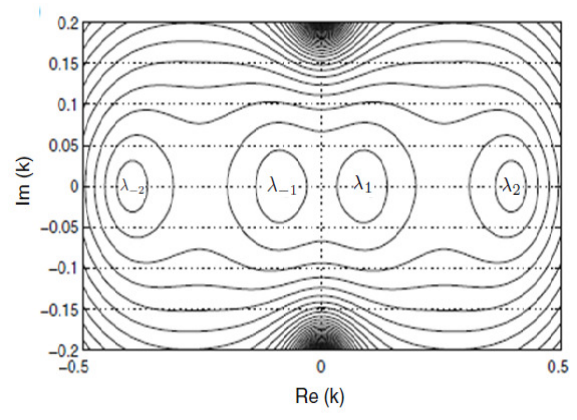

Figure 3. Contour plot of roots of the dispersion relation (2.12).

the wave numbers associated with waves propagating in the negative direction with wave numbers $\lambda_{1}$ and $\lambda_{2}$. The existence of positive real roots indicate the existence of propagating waves of two different modes. Here, it should be noted that when $\rho \rightarrow 1$ (i.e. $\rho_{1} \rightarrow \rho_{2}$ ), the problem involving scattering of waves in two-layer reduces to the problem in single layer. We have also calculated the positive real root of the relation (2.12) by taking $\rho_{1}=\rho_{2}$ and is shown in Table 1 (refer last column). In this case, i.e. when $\rho_{1}=\rho_{2}$, we have accomplished one positive real root which affirms the well known fact that the dispersion relation arises in the problem involving scattering of water waves in single layer fluid has one positive real root. In the particular case, when $\rho=1, K h=1, G h=0$ and $M / h^{2}=0$ (refer last row of Table 1 ), we have successfully obtained one positive real root which is similar to the observation made by Bora and Martha [5]. The present study further suggests that the value of both real roots increases as the value of $K h$ increases. Similar kind of observation is also noticed from Table 1 when the porosity of the bottom increases. In addition, from Table 1, it can also be seen that the values of both real roots decrease as the effect of the surface tension increases. The study reveals that the real positive roots of the dispersion relation which affect the nature of the incident wave, are sensitive to the porosity as well as the surface tension.

\subsection{Effect of various physical parameters}

Figures 4(a)-(c) depict the first order reflection and transmission coefficients due to incident wave of mode $\lambda_{1}$ for depth ratio $H / h=2$, amplitude of the sinusoidal ripple $a / h=0.1$, wave number of the ripples $\gamma h=1$, density ratio $\rho=0.5$, surface tension parameter $M / h^{2}=0.2$, porosity $G h=0.05$ and for different number of ripples (different values of $m$ and $n$ ). Similarly, in Figures 5(a)-(c) the first order reflection and transmission coefficients due to incident wave of mode $\lambda_{2}$ are shown for different number of ripples present in the undulating bottom. One common feature to all these figures (Figures 4(a)-(c) and $5(\mathrm{a})-(\mathrm{c}))$ is that they are oscillatory in nature and assume zero values for several values of $K h$ which validates the theoretical observation as described in the beginning of Section 6. Another feature which is also common to all 


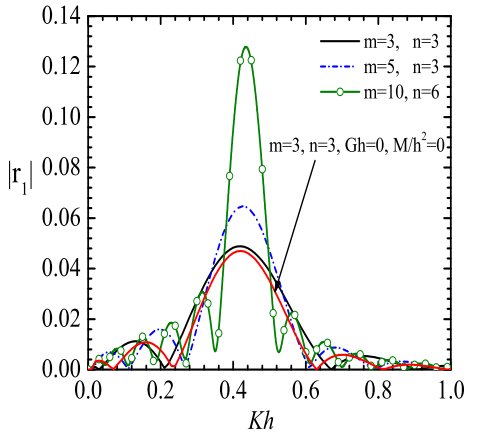

(a)

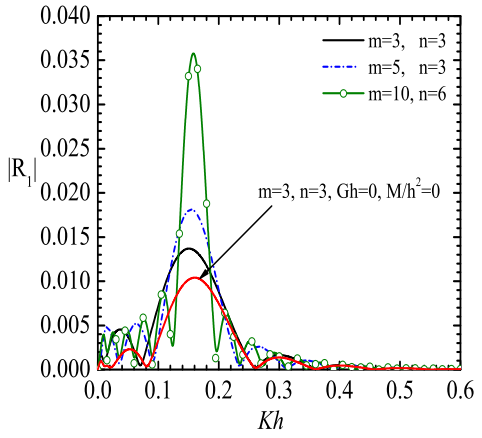

(b)

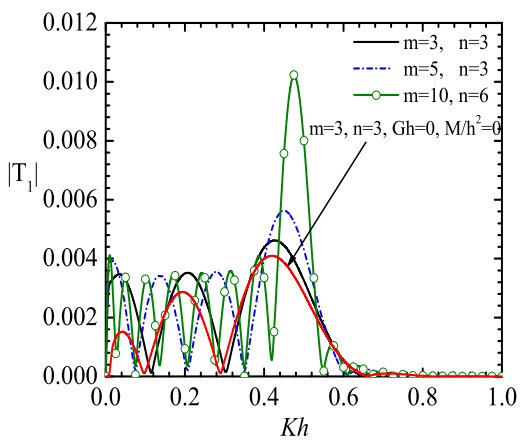

(c)

Figure 4. Coefficients due to incident wave of mode $\lambda_{1}$ in (a) $\left|r_{1}\right| ;$ (b) $\left|R_{1}\right| ;$ (c) $\left|T_{1}\right|$ for $H / h=2, a / h=0.1, \gamma h=1, \rho=0.5, M / h^{2}=0.2, G h=0.05$.

of these Figures is that both the oscillatory nature and the number of zero values increase as the number of ripple increases. The reason of the oscillatory nature is mainly due to the multiple interactions of the incident wave with the sinusoidal undulation, the interface and the free surface. Further, it is also noticed from Figure 4(a) that the peak value of $\left|r_{1}\right|$ is 0.1281 which attains when $K h=0.44$, correspondingly to $\lambda_{1}=0.501$. This confirms the theoretical observation (i.e. wave number of undulating bottom becomes approximately twice the wave number of the surface wave) described in the beginning of Section 6. It can be seen from Figure 4(b) that the highest peak value of $\left|R_{1}\right|$ is achieved at $K h=0.16$ when $\lambda_{1}=0.2918$ and $\lambda_{2}=0.7105$. In Figure 4(c), the highest peak value of $\left|T_{1}\right|$ is achieved at $K h=0.475$ when $\lambda_{1}=0.5267$ and $\lambda_{2}=1.5281$. These affirm that the peak values of $\left|R_{1}\right|$ and $\left|T_{1}\right|$ occur when $\gamma=\left(\lambda_{2}+\lambda_{1}\right)$ and $\gamma=\left(\lambda_{2}-\lambda_{1}\right)$, respectively.

For the incident wave of mode $\lambda_{2}$, a similar phenomenon is also observed from Figures 5(a)-(c). It is further noticed from Figures 4(a)-(c) and 5(a)(c) that the peak values of the reflection and transmission coefficients increase as the number of ripples increases. In certain cases, if the number of ripples 
increases indefinitely, the first order coefficients become unbounded for certain values of $K h$ and this is known as Bragg resonance [18]. In addition, the values of the coefficients $\left|t_{1}\right|$ and $\left|\widehat{T}_{1}\right|$ vanish when both $m$ and $n$ are either even or odd as it can be realized from relations (5.6) and (5.7), respectively. Therefore, these two coefficients are not shown in the figure.

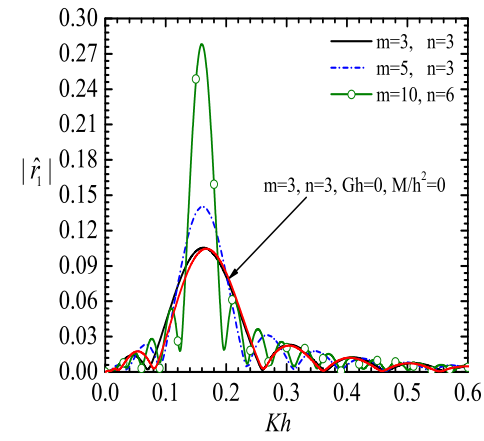

(a)

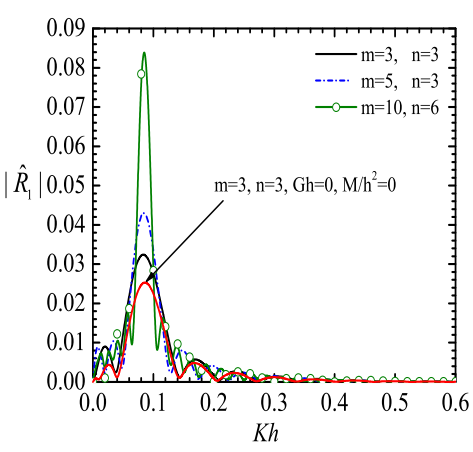

(b)

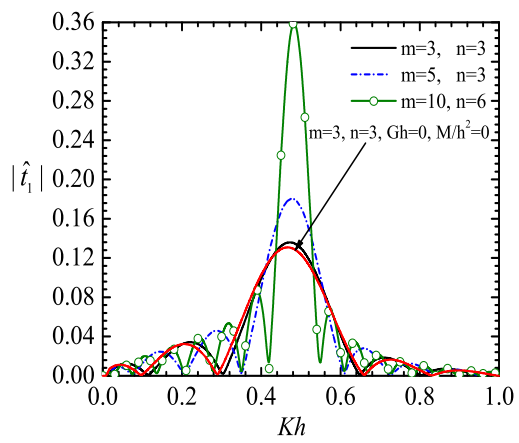

(c)

Figure 5. Coefficients due to incident wave of mode $\lambda_{2}$ in (a) $\left|\widehat{r}_{1}\right| ;$ (b) $\left|\widehat{R}_{1}\right| ;$ (c) $\left|\widehat{t}_{1}\right|$ for $H / h=2, a / h=0.1, \gamma h=1, \rho=0.5, M / h^{2}=0.2, G h=0.05$.

To compare the present results with the results available in the literature, the first order coefficients are also computed for $G h=0$ (i.e. impermeable bottom) as well as $M / h^{2}=0$ (i.e. excluding the effect of surface tension) with $m=3, n=3$ in Figures 4(a)-(c) and 5(a)-(c). From these figures, it can be noticed that the reflection and transmission in both layers due to both modes are less if the effect of the porosity and surface tension is neglected. Hence, the study reveals that the consideration of porosity as well as the effect of the surface tension is important to analyze the actual reflection and transmission phenomena in the water wave theory. Further, the study also discloses that the values of $\left|R_{1}\right|$ and $\left|\widehat{R}_{1}\right|$ are quite small as compared to the values of the 
coefficients $\left|r_{1}\right|$ and $\left|\widehat{r}_{1}\right|$ (refer Figures 4(a)-(b), 5(a)-(b)) respectively. This phenomenon indicates that the occurrence of the reflection (due to incident waves of both modes) in the upper layer is more than the lower layer.

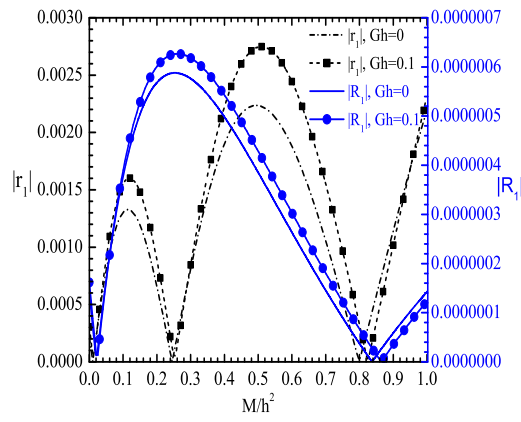

(a)

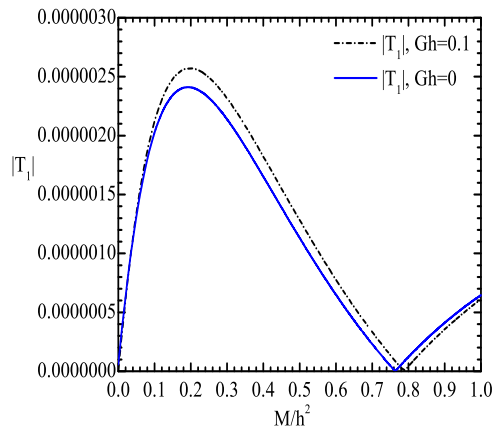

(b)

Figure 6. Coefficients due to incident wave of mode $\lambda_{1}$ in (a) $\left|r_{1}\right|$ and $\left|R_{1}\right|$; (b) $\left|T_{1}\right|$ for $\rho=0.5, H / h=2, K h=0.5, \gamma h=1, a / h=0.1, m=5, n=3$.

The effect of the surface tension on the first order reflection and transmission coefficients due to incident wave of both modes is shown in Figure 6(a)-(b) and Figure $7(\mathrm{a})-(\mathrm{b})$.

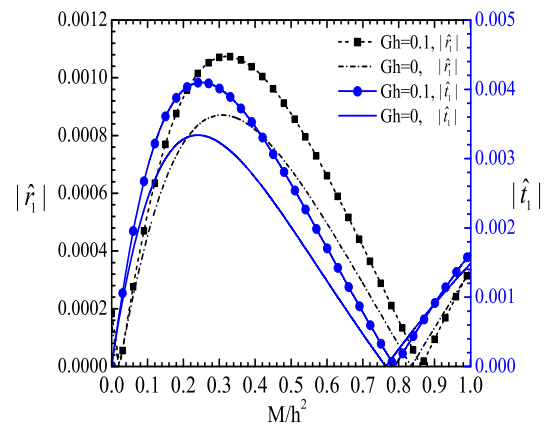

(a)

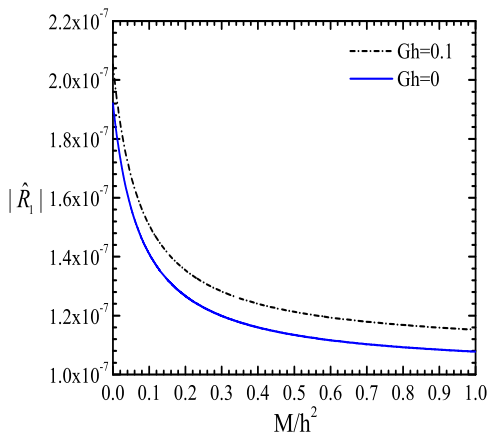

(b)

Figure 7. Coefficients due to incident wave of mode $\lambda_{2}$ in (a) $\left|\widehat{r}_{1}\right|$ and $\left|\widehat{t}_{1}\right| ;(\mathrm{b})\left|\widehat{R}_{1}\right|$ for $\rho=0.5, H / h=2, K h=0.5, \gamma h=1, a / h=0.1, m=5, n=3$.

Figure 6(a) demonstrates the first order reflection coefficients $\left|r_{1}\right|$ and $\left|R_{1}\right|$ against $M / h^{2}$ for two different values of porous effect parameter $G h=0$ and 0.1 with $\rho=0.5, H / h=2, K h=0.5, \gamma h=1, a / h=0.1, m=5$ and $n=3$. In this figure, the solid blue lines (colour available on-line) with the scale shown in right hand side axis indicate the values of the first order reflection coefficient $\left|R_{1}\right|$, whereas the dotted black lines with the scale shown in left hand side of the 
axis indicate the values of the reflection coefficient $\left|r_{1}\right|$. In Figure $6(\mathrm{~b})$, the first order transmission coefficient $\left|T_{1}\right|$ due to incident wave of mode $\lambda_{1}$ is depicted against $M / h^{2}$. When the incident wave is of mode $\lambda_{2}$, the first order reflection and transmission coefficients $\left|\widehat{r}_{1}\right|$ and $\left|\widehat{t}_{1}\right|$ are depicted in Figure $7(\mathrm{a})$ and the coefficient $\left|\widehat{R}_{1}\right|$ is depicted in Figure $7(\mathrm{~b})$. From Figures 7 and 6 , it is observed that the reflection and transmission in both layers due to incident wave of both modes are higher than those obtained for impermeable bottom $(G h=0)$. The similar phenomenon is also observed for the problem involving scattering of water waves in a single layer fluid [4]. Further, it is noticed that the peak values of the reflection and transmission coefficients due to incident wave of both modes increase up to a certain value of the surface tension parameter and after that the values start decreasing as it can be seen from Figures 6(a)-(b) and $7(\mathrm{a})$. In Figure 7(b), we have noticed that the value of reflection coefficient $\left|\widehat{R}_{1}\right|$ in the lower layer due to the incident wave of mode $\lambda_{2}$ is very small, and this is due to the effect of surface tension at the free surface of the upper layer.

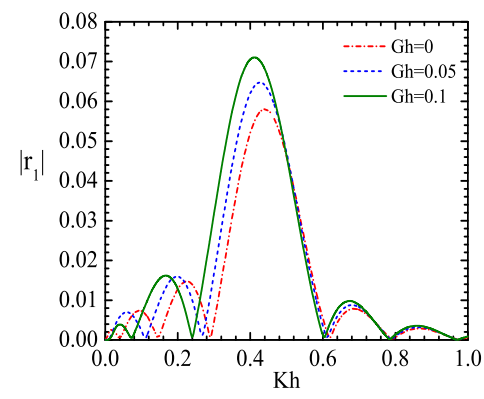

(a)

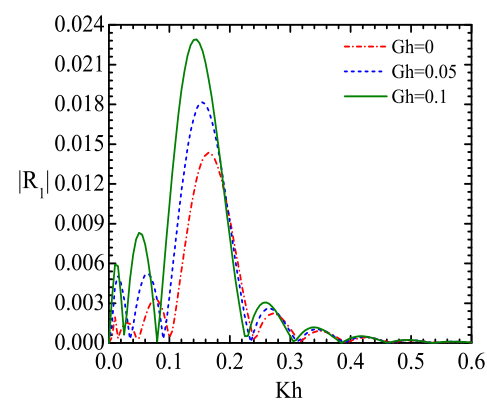

(b)

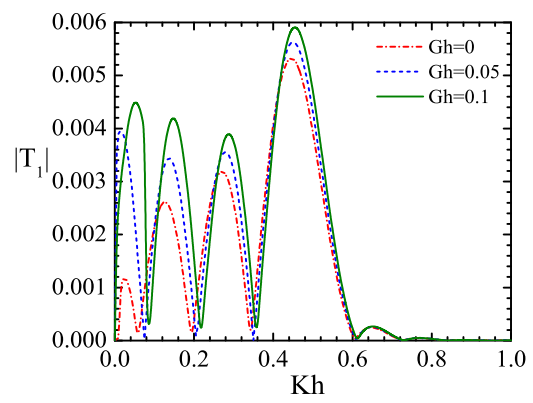

(c)

Figure 8. Coefficients due to incident wave of mode $\lambda_{1}$ in (a) $\left|r_{1}\right| ;$ (b) $\left|R_{1}\right| ;$ (c) $\left|T_{1}\right|$ for $\rho=0.5, H / h=2, M / h^{2}=0.2, \gamma h=1, a / h=0.1, m=5, n=3$.

As mentioned above, here also it is observed that the values of $\left|R_{1}\right|$ and $\left|\widehat{R}_{1}\right|$ are lower as compared to the values of the coefficients $\left|r_{1}\right|$ and $\left|\widehat{r}_{1}\right|$ respectively (see, Figures 6(a), 7(a) and $7(\mathrm{~b})$ ). The above observations reveal that the 
effect of surface tension can be neglected (although the surface tension effects the transformation of incident wave energy between the waves of two modes) in practice as the results presented in Figures 7 and 6 are smaller than one percent.

The outcome of the effect of porosity on the reflection and transmission coefficients is demonstrated in Figures 8(a)-(c) and 9(a)-(c).

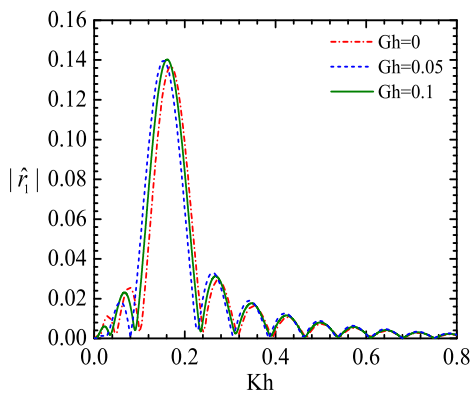

(a)

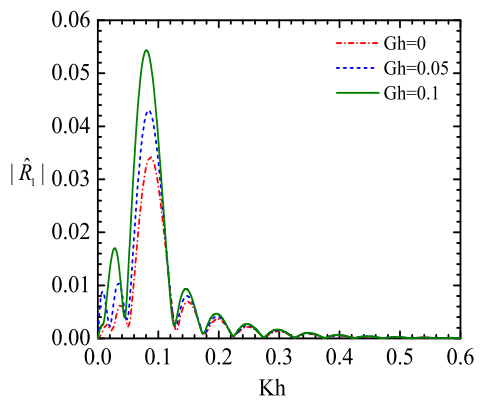

(b)

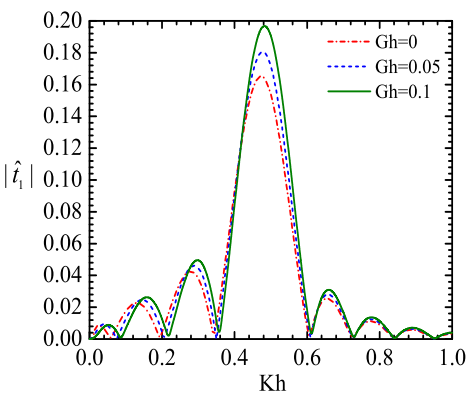

(c)

Figure 9. Coefficients due to incident wave of mode $\lambda_{2}$ in (a) $\left|\widehat{r}_{1}\right| ;$ (b) $\left|\widehat{R}_{1}\right| ;$ (c) $\left|\widehat{t}_{1}\right|$ for $\rho=0.5, H / h=2, M / h^{2}=0.2, \gamma h=1, a / h=0.1, m=5, n=3$.

In Figures 8(a)-(c), the first order reflection and transmission coefficients due to the incident wave of mode $\lambda_{1}$ are demonstrated for three different values of $G h=0,0.05$ and 0.1 with $\rho=0.5, H / h=2, M / h^{2}=0.2, \gamma h=1, a / h=$ $0.1, m=5$ and $n=3$, whereas Figures $9(\mathrm{a})-(\mathrm{c})$ demonstrate the same due to the incident wave of mode $\lambda_{2}$ for $G h=0,0.05$ and 0.1 . The other two transmission coefficients $t_{1}$ and $\widehat{T}_{1}$ are not shown as their values are assumed to be zero due to the consideration of odd values of $m$ and $n$. It is noticed from Figures 8(a)-(c), the values of all coefficients due to the incident wave of mode $\lambda_{1}$ gradually increase as the value of the porosity increase. Similar kind of nature was also found by Martha et al. [4] who analyzed scattering of water waves by an undulating permeable bottom in a single layer fluid. The above phenomenon can also be realized from relations (5.3)-(5.5) as the 
values of the numerators in the expressions of the coefficients increase and the denominators decrease with increase of the porous effect parameter $G$. In the case of incident wave of mode $\lambda_{2}$, a similar kind of observation is also noticed as can be seen from Figures $9(\mathrm{a})-(\mathrm{c})$. It is worth mentioning that the more reflection occurs in the upper layer as compared to the lower layer which can be seen from Figures $8(\mathrm{a})$ and $8(\mathrm{~b})$. This may be due to the interaction of water waves between the surface mode and the internal mode in a two-layer fluid. From Figures $9(\mathrm{a})-(\mathrm{b})$, it is observed that the reflection coefficients $\left|\widehat{r}_{1}\right|$ and $\left|\widehat{R}_{1}\right|$ due to the incident wave of mode $\lambda_{2}$, demonstrate the similar kind of nature respectively as noticed above in the case of incident wave of mode $\lambda_{1}$. These observations indicate that the porosity is quite sensitive to the reflection and transmission coefficients due to incident wave of both modes.

\subsection{Free surface elevation and interface elevation}

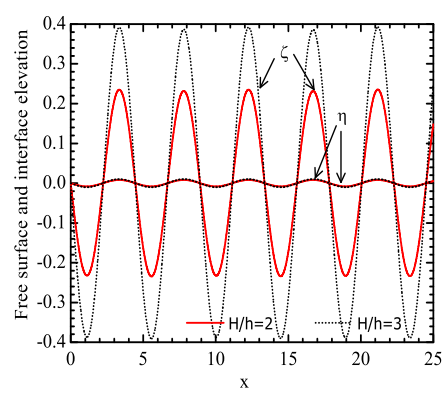

(a)

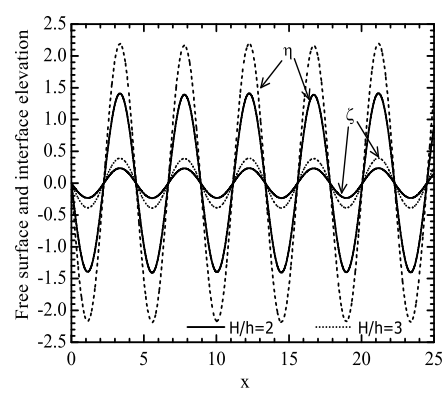

(b)

Figure 10. Variation of free surface elevation $\eta$ and interface elevation $\zeta$ for various values of depth ratios $H / h$ : (a) waves with surface mode and (b) waves with interface mode.

In this subsection, the behaviours of the free surface elevation $(\eta)$ and interface elevation $(\zeta)$ are discussed (refer Figures 10 and 11). The free surface elevation $\eta$ and interface elevation $\zeta$ are determined from the linearized free surface dynamic condition and interface dynamic condition, respectively. In Figure 10(a)-(b), the variation of the free surface elevation $\eta$ and interface elevation $\zeta$ are plotted for various values of depth ratios $H / h$. It can be noticed from Figure 10 that, when the incident wave is of surface mode, the disturbance at the free surface is larger in amplitude as compared to the disturbance occurs at the interface. But, when the incident wave of interface mode, the disturbance occurs at the free surface is not significant. However, the variation of the interface elevation is significant as can be seen from Figure 10(b). A similar kind of phenomenon is also observed in [12]. Figure 10 also suggests that the disturbance (in terms of amplitude) at the free surface as well as at the interface increases with the depth ratio. Here it is worthy to note that the increment in the amplitude of the free surface profile due to incident wave with interface mode is negligible (refer Figure 10(b)). This indicates that the 
free surface profile is not affected too much by the incident wave with interface mode.

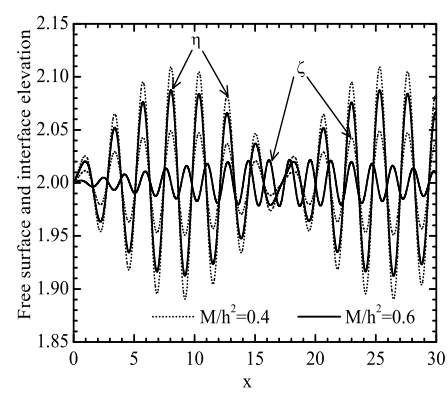

(a)

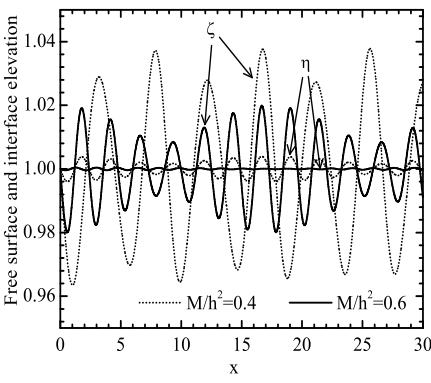

(b)

Figure 11. Variation of free surface elevation $\eta$ and interface elevation $\zeta$ for various values of surface tension effect parameter $M / h^{2}$ : (a) waves with surface mode and (b) waves with interface mode.

In Figure 11(a)-(b), the variations of free surface elevation and interface elevation are demonstrated due to incident wave of surface mode and interface mode, respectively, for various values of surface tension effect parameter $M / h^{2}$. It can be observed from this figure that the surface tension parameter effects the free surface elevation as well as the interface elevation. When the incident wave is of surface mode, the free surface as well as the interface profiles are greatly affected. But, when the incident wave is of interface mode, the free surface profile is not affected too much (refer Figure 11(b)). This is due to the fact that the free surface, which is situated above the interface, is far away from the interface. It is also observed (refer Figure 11) that the disturbance (in term of amplitude of the profile) decreases as the surface tension increases. This agrees the physical intuition that the increment in the surface tension decreases the disturbance/variation of the profiles.

\subsection{Energy identities}

Out of many important results in the study of scattering of water waves, the special relation known as "energy balance relation or energy identity" plays a very important role for checking the method of solution of mixed boundary value problem arises in water wave theory. The energy identity [6] relates the reflection as well as transmission coefficients associated with the scattering problem, which supports the theoretical validation of the present problem in the absence of experimental evidences. In a two-layer fluid system, the presence of surface tension leads to two distinct energy identities corresponding to incident waves of both wave numbers.

The energy balance relations is derived using modified Green's integral theorem. When the incident wave is of wave number $\lambda_{1}$, we have derived the 
energy balance relation as

$$
|r|^{2}+|t|^{2}+J\left(|R|^{2}+|T|^{2}\right)=1
$$

where $J=J_{\lambda_{2}} / J_{\lambda_{1}}$ with

$$
J_{k}=i\left[M k^{3}+k\right] \times\left[\rho \int_{-h}^{0}\left(f_{1}(k, y)\right)^{2} d y+\int_{0}^{H}\left(f_{2}(k, y)\right)^{2} d y\right], \quad k=\lambda_{1}, \lambda_{2}
$$

Similarly, when the incident wave is of wave number $\lambda_{2}$, the energy balance relation can be derived as

$$
\frac{1}{J}\left(|\widehat{r}|^{2}+|\widehat{t}|^{2}\right)+|\widehat{R}|^{2}+|\widehat{T}|^{2}=1
$$

For ensuring the correctness of the computed numerical results, the energy balance relations (6.1) and (6.2) associated with the present problem are checked. For incident wave of mode $\lambda_{1}$, we have presented the variation of $|r|,|R|,|t|,|T|$ and $|r|^{2}+|t|^{2}+J\left(|R|^{2}+|T|^{2}\right)$ (left hand side of the energy balance relation (6.1)) for various values of $K h$ in Table 2 along with other

\begin{tabular}{|c|c|c|c|c|c|}
\hline \multirow{2}{*}{$K h$} & \multicolumn{4}{|c|}{ Values of coefficients } & \multirow{2}{*}{$|r|^{2}+|t|^{2}+J\left(|R|^{2}+|T|^{2}\right)$} \\
\hline & $|r|$ & $|R|$ & $|t|$ & $|T|$ & \\
\hline 0.2 & $6.2555 \mathrm{e}-03$ & $4.0775 \mathrm{e}-03$ & 1.0000 & $2.6514 \mathrm{e}-04$ & 1.0002 \\
\hline 0.3 & $4.9253 \mathrm{e}-03$ & $4.7323 \mathrm{e}-04$ & 1.0000 & $1.3875 \mathrm{e}-03$ & 1.0000 \\
\hline 0.4 & $2.2350 \mathrm{e}-02$ & $5.6159 \mathrm{e}-05$ & 1.0000 & $1.6341 \mathrm{e}-03$ & 1.0006 \\
\hline 0.6 & $5.5462 \mathrm{e}-03$ & $1.9753 \mathrm{e}-05$ & 1.0000 & $4.9459 \mathrm{e}-06$ & 1.0000 \\
\hline 0.8 & $1.6625 \mathrm{e}-03$ & $2.6479 \mathrm{e}-06$ & 1.0000 & 7.3963e-06 & 1.0000 \\
\hline 1 & $8.2468 \mathrm{e}-04$ & $2.0775 \mathrm{e}-07$ & 1.0000 & $6.9394 \mathrm{e}-07$ & 1.0000 \\
\hline
\end{tabular}
parameters as $m=5, n=3, \varepsilon / h=0.4, M / h^{2}=0.2, G h=0.05$ and $\rho=0.5$.

Table 2. Values of all coefficients for energy identity relation (6.1)

The last column of the Table 2 shows that each value of $|r|^{2}+|t|^{2}+$ $J\left(|R|^{2}+|T|^{2}\right)$ for each $K h$ is nearly equal to 1 , satisfying the energy balance relation accurately. Similarly, the variation of $|\widehat{r}|,|\widehat{R}|,|\widehat{t}|,|\widehat{T}|$ and $\frac{1}{J}\left(|\widehat{r}|^{2}+\right.$ $\left.|\widehat{t}|^{2}\right)+|\widehat{R}|^{2}+|\widehat{T}|^{2}$ are shown in Table 3 for different values of $K h$ along with the same set of values of the parameters listed for Table 2.

The last column of Table 3 reflects that each value of left hand side of relation (6.2) is nearly equal to 1 for each $K h$ which fulfill the energy balance relation (6.2). For more convenient, two plots: (i) $|r|^{2}+|t|^{2}+J\left(|R|^{2}+|T|^{2}\right)$ versus $K h$ and (ii) $\frac{1}{J}\left(|\widehat{r}|^{2}+|\widehat{t}|^{2}\right)+|\widehat{R}|^{2}+|\widehat{T}|^{2}$ versus $K h$ have been demonstrated in Figure 12(a)-(b). From the results shown in Figures 12(a)-(b) and Tables 2 and 3 , it can be concluded that the results derived in the present paper are accurate. 
Table 3. Values of all coefficients for energy identity relation (6.2)

\begin{tabular}{|c|c|c|c|c|c|}
\hline \multirow{2}{*}{$K h$} & \multicolumn{4}{|c|}{ Values of coefficients } & \multirow{2}{*}{$\frac{1}{J}\left(|\widehat{r}|^{2}+|\widehat{t}|^{2}\right)+|\widehat{R}|^{2}+|\widehat{T}|^{2}$} \\
\hline & $|\widehat{r}|$ & $|\widehat{R}|$ & $|\widehat{t}|$ & $|\widehat{T}|$ & \\
\hline 0.2 & $3.5270 \mathrm{e}-02$ & $1.7289 \mathrm{e}-03$ & $2.2934 \mathrm{e}-03$ & 1.0000 & 1.00005 \\
\hline 0.4 & $1.1818 \mathrm{e}-03$ & $2.5511 \mathrm{e}-04$ & $3.5186 \mathrm{e}-02$ & 1.0000 & 1.00082 \\
\hline 0.6 & $1.4739 \mathrm{e}-03$ & $2.4553 \mathrm{e}-05$ & $3.6904 \mathrm{e}-04$ & 1.0000 & 1.00002 \\
\hline 0.8 & $9.0178 \mathrm{e}-04$ & $3.0326 \mathrm{e}-06$ & $2.5189 \mathrm{e}-03$ & 1.0000 & 1.00003 \\
\hline 1 & $3.6663 \mathrm{e}-04$ & $4.7978 \mathrm{e}-08$ & $1.2247 \mathrm{e}-03$ & 1.0000 & 1.00000 \\
\hline
\end{tabular}

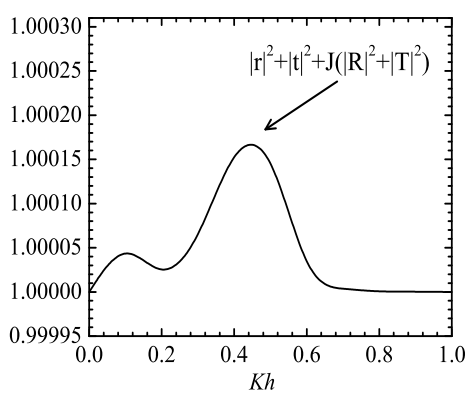

(a)

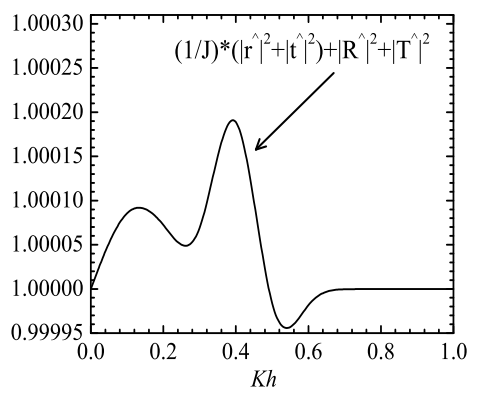

(b)

Figure 12. (a) $|r|^{2}+|t|^{2}+J\left(|R|^{2}+|T|^{2}\right)$ versus $K h$, and (b) $\frac{1}{J}\left(|\widehat{r}|^{2}+|\widehat{t}|^{2}\right)+|\widehat{R}|^{2}+|\widehat{T}|^{2}$ versus $K h$ for $\rho=0.5, H / h=2, M / h^{2}=0.2$.

\section{Conclusions}

The study analyzes the reflection and transmission phenomena involved in water wave scattering by undulating porous bottom in a two-layer fluid system in the presence of surface tension. Based on the linear water wave theory, the physical problem is formulated as a coupled boundary value problem. The inclusion of surface tension gives rise to a third order boundary condition which makes the governing boundary value problem more complicated. Perturbation analysis in conjunction with Fourier transform technique is employed to derive the velocity potentials, first order reflection and transmission coefficients. The main advantage of Fourier transform method is that we need to solve relatively easier ordinary differential equations to find out the velocity potentials. It is noticed that the reflection and transmission coefficients depend on the shape as well as the porosity of the undulating bottom. Hence, two different kinds of undulations are considered to determine the reflection and transmission. From the practical point of view, the reflection and transmission phenomenon for sinusoidal bottom topography is presented graphically to demonstrate how the wave energy is transferred from one mode to another mode. Role of various system parameters such as number of ripples present in the undulating bottom, surface tension and porous effect parameters are also discussed. It is observed 
that the reflection and transmission coefficients are oscillatory in nature and the peak values of these coefficients increases as the number of ripples increases. The study also reveals that the reflection and transmission in both modes increase when the porosity of the bottom increases. Reflection and transmission in both layers due to incident wave of both modes are less if the effect of the porosity and surface tension is neglected. It is also noticed that the more energy is transferred by the reflected wave of surface mode than the reflected wave of interface mode. In the present study, the behaviours of the free surface elevation and interface elevation are discussed.

\section{References}

[1] R. Harter, I.D. Abrahams and M.J. Simon. The effect of surface tension on trapped modes in water-wave problems. Proc. R. Soc. A, 463:3131-3149, 2007. https://doi.org/10.1098/rspa.2007.0063.

[2] H. Mase, S.I. Oki and K. Takeba. Wave equation over permeable rippled bed and analysis of bragg scattering of surface gravity waves. J. Hydraul. Res., 33(6):789-812, 1995. https://doi.org/10.1080/00221689509498552.

[3] G.S. Beavers and D.D. Joseph. Boundary conditions at a naturally permeable wall. J. Fluid Mech., 30(1):197-207, 1967. https://doi.org/10.1017/S0022112067001375.

[4] S.C. Martha, S.N. Bora and A. Chakrabarti. Oblique water-wave scattering by small undulation on a porous sea-bed. Appl. Ocean Res., 29:86-90, 2007. https://doi.org/10.1016/j.apor.2007.07.001.

[5] S.N. Bora and S.C. Martha. Scattering of surface waves over an uneven sea-bed. Appl. Math. Lett., 21(10):1082-1089, 2008. https://doi.org/10.1016/j.aml.2007.12.005.

[6] A. Chakrabarti and S. Mohapatra. Scattering of surface water waves involving semi-infinite floating elastic plates on water of finite depth. J. Mar. Sci. Appl., 12(3):325-333, 2013. https://doi.org/10.1007/s11804-013-1204-z.

[7] A. Chakrabarti and T. Sahoo. The effect of surface tension in porous wave maker problems. J. Austral. Math. Soc. Ser. B, 39(4):539-556, 1998. https://doi.org/10.1017/S0334270000007797.

[8] P.G. Chamberlain and D. Porter. Wave scattering in a twolayer fluid of varying depth. J. Fluid Mech., 524:207-228, 2005. https://doi.org/10.1017/S0022112004002356.

[9] A.T. Chwang. A porous-wavemaker theory. J. Fluid Mech., 132:395-406, 1983. https://doi.org/10.1017/S0022112083001676.

[10] A.G. Davies. The reflection of wave energy by undulation on the seabed. Dynam. Atmos. Oceans., 6(4):207-232, 1982. https://doi.org/10.1016/03770265(82)90029-X.

[11] A.G. Davies and A.D. Heathershaw. Surface-wave propagation over sinusoidally varying topography. J. Fluid Mech., 144:419-443, 1984. https://doi.org/10.1017/S0022112084001671.

[12] S.C. Mohapatra, D. Karmakar and T. Sahoo. On capillary gravitywave motion in two-layer fluids. J. Eng. Math., 71(3):253-277, 2011. https://doi.org/10.1007/S10665-011-9451-y. 
[13] H. Behera, S. Koley and T. Sahoo. Wave transmission by partial porous structures in two-layer fluid. Eng. Anal. Bound. Elem., 58:58-78, 2015. https://doi.org/10.1016/j.enganabound.2015.03.010.

[14] C.M. Linton and J.R. Cadby. Scattering of oblique waves in a two-layer fluid. J. Fluid Mech., 461:343-364, 2002. https://doi.org/10.1017/S002211200200842X.

[15] C.M. Linton and M. McIver. The interaction of waves with horizontal cylinders in a two-layer fluids. J. Fluid Mech., 304:213-229, 1995. https://doi.org/10.1017/S002211209500440X.

[16] P. Maiti and B.N. Mandal. Scattering of oblique waves by bottom undulations in a two-layer fluid. J. Appl. Math. Comput., 22(3):21-39, 2006. https://doi.org/10.1007/BF02832035.

[17] P. Maiti and B.N. Mandal. Water wave scattering by an elastic plate floating in an ocean with a porous bed. Appl. Ocean Res., 47:73-84, 2014. https://doi.org/10.1016/j.apor.2014.03.006.

[18] C.C. Mei. Resonant reflection of surface waves by periodic sandbars. J. Fluid Mech., 152:315-335, 1985. https://doi.org/10.1017/S0022112085000714.

[19] S. Mohapatra and S.N. Bora. Propagation of oblique waves over small bottom undulation in an ice-covered two-layer fluid. Geophys. Astrophys. Fluid Dyn., 103(5):347-374, 2009. https://doi.org/10.1080/03091920903071077.

[20] S. Mohapatra and S.N. Bora. Reflection and transmission of water waves in a two-layer fluid flowing through a channel with undulating bed. Z. Angew. Math. Mech., 91:46-56, 2011. https://doi.org/10.1002/zamm.200800216.

[21] S. Panda. Oblique wave scattering by an undulating porous bottom in a two-layer ice-covered fluid. China Ocean Eng., 30(3):431-446, 2015. https://doi.org/10.1007/s13344-016-0067-x.

[22] S. Panda and S.C. Martha. Interaction of water waves with small undulations on a porous bed in a two-layer ice-covered fluid. J. Mar. Sci. Appl., 12(4):381-392, 2013. https://doi.org/10.1007/s11804-013-1208-8.

[23] S. Panda and S.C. Martha. Oblique wave scattering by undulating porous bottom in a two-layer fluid: Fourier transform approach. Geophys. Astrophys. Fluid Dyn., 108(6):587-614, 2014. https://doi.org/10.1080/03091929.2014.953948.

[24] P.F. Rhodes-Robinson. The effect of surface tension on the progressive waves due to incomplete vertical wave-makers in water of infinite depth. Proc. R. Soc. Lond. Ser. A, 435:293-319, 1991. https://doi.org/10.1098/rspa.1991.0146.

[25] S. Panda, S.S. Samantaray and S.C. Martha. Wave scattering by small undulation on the porous bottom of an ocean in the presence of surface tension. ISRN Oceanography, 2013:1-6, 2013. https://doi.org/10.5402/2013/504879. 